\title{
Total Joint Arthroplasty in Patients with Inflammatory Rheumatic Diseases
}

\author{
Riccardo Compagnoni · Roberta Gualtierotti · Pietro Randelli
}

Received: June 19, 2018 / Published online: July 11, 2018

(C) The Author(s) 2018

\section{ABSTRACT}

Since its introduction, total joint arthroplasty (TJA) has improved the quality of life of patients with degenerative joint disorders. In the last decades, a number of conventional and biological disease-modifying antirheumatic drugs have become available for the treatment of patients with inflammatory rheumatic diseases (IRD), leading to a reduction in the need to undergo TJA. However, TJA is still frequently performed in IRD patients. Both rheumatologists and orthopedics should be aware that

Enhanced digital features To view enhanced digital features for this article go to https://doi.org/10.6084/ m9.figshare.6714140.

R. Compagnoni $(\bowtie) \cdot$ P. Randelli

Clinica Ortopedica, ASST Centro Specialistico

Ortopedico Traumatologico Gaetano Pini-CTO, Milan, Italy

e-mail: riccardo.compagnoni@gmail.com

R. Compagnoni · P. Randelli

Laboratory of Applied Biomechanics, Department of Biomedical Sciences for Health, Università degli

Studi di Milano, Milan, Italy

R. Gualtierotti

Dipartimento di Reumatologia e Scienze Mediche, ASST Centro Specialistico Ortopedico

Traumatologico Gaetano Pini-CTO, Milan, Italy

R. Gualtierotti

Department of Clinical Sciences and Community

Health, Università degli Studi di Milano, Milan, Italy patients with IRD have a peculiar perioperative risk profile due to disease-related, patient-related, and surgery-related risk factors. On the basis of current evidence, TJA is a safe procedure for IRD patients as long as an accurate risk stratification and a multidisciplinary approach are applied. We here describe the current strategies for an appropriate surgical management of osteoarthritis in IRD patients and the fascinating opening perspectives that surgeons and clinicians may expect in the future.

Keywords: Inflammatory rheumatic diseases; Psoriatic arthritis; Rheumatoid arthritis; Rheumatology; Systemic lupus erythematosus; Total joint arthroplasty

\section{INTRODUCTION}

Orthopedic surgery in patients with inflammatory rheumatic diseases (IRD) historically represents a challenge for clinicians and orthopedic surgeons. The prevalence of IRD ranges from $0.05 \%$ to $1 \%$ in the general population, with many patients that need a surgical treatment such as total joint arthroplasty (TJA) because of articular degeneration $[1,2]$. Since the introduction of corticosteroids and non-steroidal anti-inflammatory drugs (NSAIDs), in the 1950s, it was clear that this approach resulted in multiple side effects without affecting disease 
progression. Over the last decades, the introduction of conventional synthetic diseasemodifying antirheumatic drugs (csDMARDs) such as methotrexate and the acknowledgement of the importance of early diagnosis, prompt treatment, and treat-to-target approach have led to the improvement of IRD management. The development and introduction in clinical practice of biological DMARDs (bDMARDs) and, more recently, of targeted synthetic DMARDs (tsDMARDs) such as tofacitinib have dramatically changed the prognosis of patients with RA (Fig. 1). The modern treatment of IRD has reduced the need for TJA, but this procedure is still frequently performed and a multidisciplinary approach is required to reduce the risk of adverse events [3]. Surgical treatment in patients with IRD is often debated among surgeons because of the fear of infections, disease flares, and negative surgical outcomes. In patients with IRD three sets of specific medical aspects need to be considered when the articular degeneration requires TJA: the first set is related to the underlying disease, the second set is related to therapies, and the third is related to surgical technique. Correct management results in good surgical outcomes and a substantial decrease in the number of adverse events. The aim of this communication is to describe the current strategies for appropriate management of osteoarthritis in IRD patients and the fascinating opening perspectives that surgeons and clinicians may expect for new drugs and surgical techniques.

\section{ASPECTS RELATED TO THE UNDERLYING DISEASE}

Infections are a dreaded complication of TJA, resulting in long pharmacological therapies and worse clinical outcomes. A higher disease activity has been associated with a higher probability of developing infections. Despite the elevated risk of infection, there is currently no evidence supporting the use of perioperative protocols different from the general population, but specific attention is recommended in postoperative follow-up and wound healing [4-6]. An interesting aspect is the fear of infection related to previous corticosteroid injections. Recent comforting data from systematic reviews

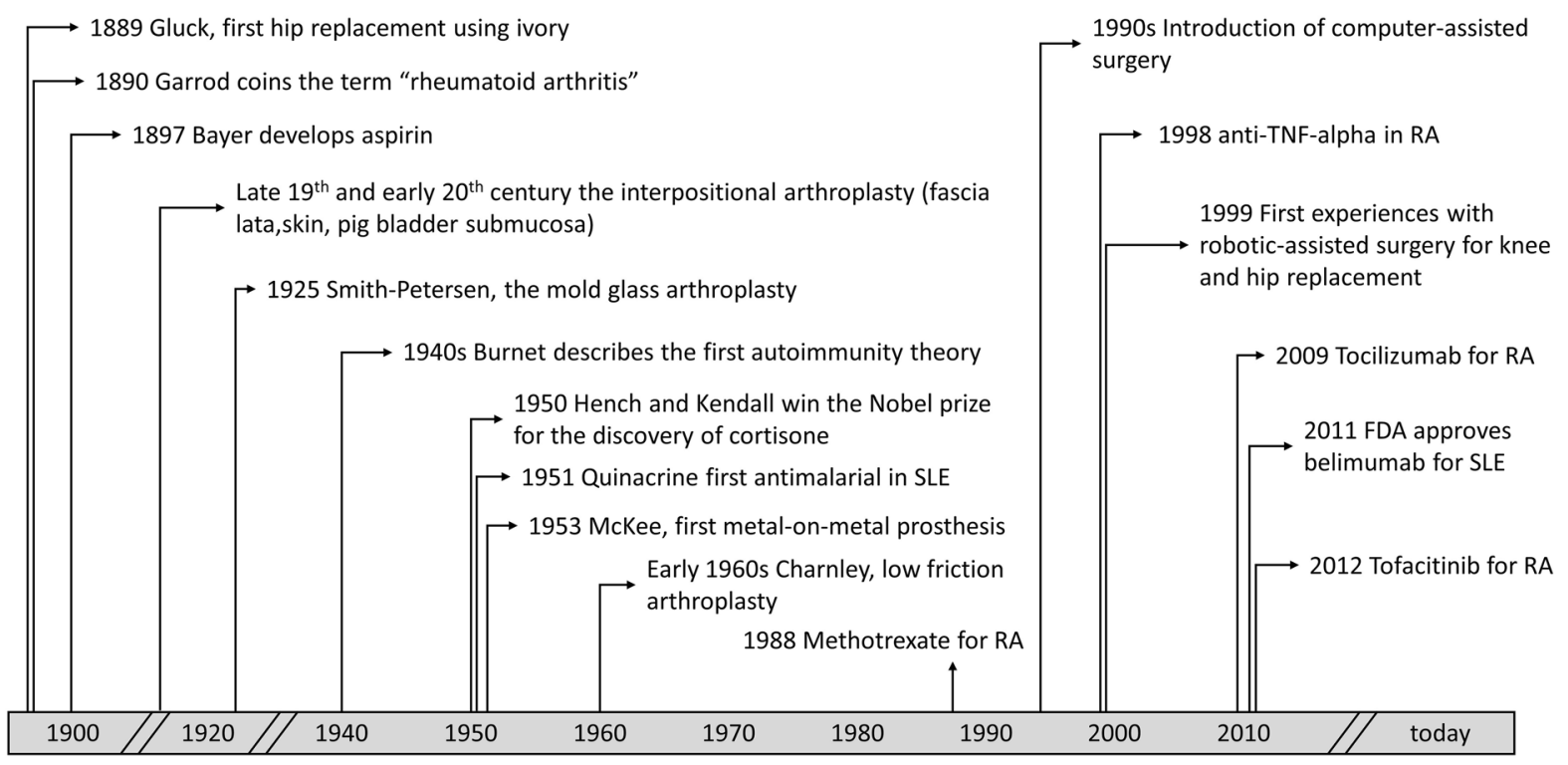

Fig. 1 Timeline of the main discoveries and inventions in orthopedics and rheumatology in the nineteenth and twentieth century. FDA Food and Drug Administration, RA rheumatoid arthritis, SLE systemic lupus erythematosus, TNF tumor necrosis factor. Modified from Mocsai et al. (2014) with permission [43] 
confirm that this fear is not actually supported by evidence [7].

Patients with IRD also have a higher risk of experiencing cardiovascular events compared to the general population $[8,9]$. The preoperative assessment of patient-related medical risk factors should include obesity, malnutrition, hyperglycemia, uncontrolled diabetes mellitus, chronic renal failure, smoking, and alcohol abuse [10]. These factors have proved to contribute to the risk of postoperative infections following TJA in IRD patients [11]. Conventional risk factors are more common in RA but do not fully explain the increased cardiovascular risk, which seems specifically related to chronic inflammation that may lead, among other mechanisms, to the reduction of high density lipoprotein levels and oxidation of low density lipoproteins with pro-inflammatory effects [12]. Considering the low risk of lifethreatening bleeding with aspirin, if no major contraindications are present, the maintenance of aspirin is feasible and recommended in all IRD patients who are already in treatment [13].

Recently, a multi-biomarker disease activity (MBDA) algorithm has been developed combining 12 biomarkers of disease activity among which are pro-inflammatory cytokines and other protein biomarkers implicated in the pathophysiology of joint disease in RA [14]. Higher MBDA scores are associated with a higher risk of infections, myocardial infarction, and coronary heart disease [15]; therefore, the use of MBDA score to stratify patients in a perioperative setting in the future could replace the assessment of CRP levels and erythrocyte sedimentation rate. Similarly, in SLE, a combined soluble mediator score incorporating 52 analytes was studied in order to improve prediction of SLE flares [16].

A higher risk of venous thromboembolism (VTE) has also been reported as a consequence of chronic inflammation and endothelial activation, which increase tissue factor expression and inhibit endogenous anticoagulants and fibrinolysis [17]. Major orthopedic procedures, especially in the lower limb, represent a wellknown risk factor for VTE in the weeks following surgery, and a delicate balance between thrombotic and bleeding events has to be found in IRD patients $[18,19]$. In preoperative cardiovascular risk stratification of IRD patients, the presence of antiphospholipid antibodies (aPL) should also be determined [20]. However, it is known that a subset of patients with persistent aPL positivity do not ever develop VTE events in their lifetime. Antibodies directed against domain I of $\beta 2$-glycoprotein I (GPI) highly correlate with thrombosis, supporting the hypothesis that these antibodies are a clinically relevant subset of anti- $\beta 2$ GPI antibodies associated with antiphospholipid syndrome (APS) [21-23]. In the future, these antibodies may be used as routine biomarkers in perioperative risk stratification. Practical management strategies to prevent potential perioperative complications in patients already treated with anticoagulants should include ensuring correct hydration of the patient, reducing to a minimum the periods without anticoagulant drugs, and encouraging postoperative mobilization as early and as much as tolerated, together with the use of antithrombotic stockings, if not contraindicated $[6,24]$.

Although the role of periodontal disease as a risk factor for periprosthetic joint infection is still controversial [25], there is an established association between periodontal disease and IRD and future studies should address the importance of an odontostomatologic evaluation prior to TJA [26]. IRD patients have a higher risk of depression and other psychological diseases due to high tension and low selfesteem followed by the perceived impact of RA, fatigue, passive coping, pain, and physical disability [27]. Considering the relationship between personality psychology and expected clinical outcomes, counselling with a psychological team before surgery is recommended [28]. Future studies should also evaluate nonpharmacological management of these patients.

Finally, models for risk stratification will become more and more accurate and personalized thanks to the most recent technologies aimed at analyzing large volumes of data sets regarding patient characteristics and surgical procedures, with the possibility to share data among different healthcare institutions [29]. 


\section{ASPECTS RELATED TO THERAPIES}

The higher risk of infection in the perioperative period in IRD patients could be associated with the underlying treatment [24]. Evidence from RA studies demonstrates that patients on bDMARDs (both TNF inhibitors and non-TNF biologics) have an increased risk of serious infections compared with patients on csDMARDs, with no differences across bDMARDs [30]. However, the risk of flares in the postsurgical period should be carefully evaluated in IRD patients because a disease flare may require a rise in corticosteroid dosage, thus increasing the risk of infection. Traditionally, a suspension of bDMARDs of at least two halflives has been recommended so far. However, half-life may not correspond to the duration of the immunosuppressant effect as much as the dosing cycle. Therefore, current guidelines recommend to withhold biologics on the basis of the dose interval and tofacitinib, a tsDMARD targeting Janus kinase/signal transducer and activator of transcription (JAK/STAT) signaling, 7 days before surgery, respectively [24]. A very recent case series of Japanese patients with RA treated with tofacitinib undergoing orthopedic surgery has been published [31]. No serious perioperative infections were reported in any patient during a follow-up of at least 6 months, thus confirming the safety of the drug.

As a result of the low risk of infection, current evidence supports the view that continuing csDMARDs such as methotrexate, sulfasalazine, hydroxychloroquine, and azathioprine is a safe option in RA patients without relevant comorbidities [32]. The use of corticosteroids with a dosage above $15 \mathrm{mg}$ daily is associated with a higher risk of infection. On the basis of current evidence, it is advisable to carefully taper the dose of corticosteroids to below $20 \mathrm{mg}$ prednisone daily and to abandon the practice of giving "stress dose steroids" because there is no evidence of a reduced risk of adrenal insufficiency against a possible increased risk of infection [24]. The use of NSAIDs should be evaluated in IRD patients in the perioperative period, because it is related to an increased cardiovascular risk and may affect wound and bone healing in association with corticosteroids and DMARDs [33]. An effective alternative in the days after surgery could be the association of paracetamol with opioids [34].

Inherited differences in drug metabolism dramatically affect drug response and adverse effects in each subject. It is tempting to hypothesize that decreasing costs of genome sequencing and growing awareness of the association between specific gene polymorphisms and drug responses will lead in the near future to evidence-based generated data that can be applied in perioperative care, in particular for pain and anesthetic drug management, but also for antirheumatic drugs [35-37].

\section{ASPECTS RELATED TO SURGERY}

TJA in IRD is a challenge for surgeons, whose responsibility is to choose the most effective and less invasive technique for these patients [38]. Many new opportunities are currently available, such as computer-assisted surgery, robotic surgery, patient-specific instrumentations, and fast-track rehabilitation protocols. Computer-assisted surgery was introduced in clinical practice at the end of 1990s, and its clinical application can potentially have a specific advantage in IRD patients. TJA performed with this technique does not require intramedullary guides, reducing blood loss and local trauma [39]. These advantages have been also described in patient-specific instrumentations [40]. Robotic surgery represents for many aspects the future of TJA. It theoretically combines the advantages of computer navigation with the robotic control of all the surgical steps regarding the bone cuts. This technique has been introduced only few years ago and the preliminary studies report good results, but actually insufficient literature has been published regarding clinical and biomechanical outcomes [41]. Fast-track rehabilitation protocols represent the state of the art of postoperative care in patients undergoing total joint replacement, and combined with perioperative enhanced recovery after surgery (ERAS) protocols can give to patients an early recovery and better clinical outcomes, especially in the first 
postoperative months as reported by many studies [42].

\section{CONCLUSIONS}

The management of DMARDs and other drugs in IRD patients undergoing TJA is debated among orthopedic surgeons and rheumatologists, thus leading to uncertainty in decisionmaking. TJA has a pivotal role in the management of IRD patients and quality of life improvement. Overall, evidence supports the view that TJA can be performed safely in IRD patients, as long as a careful risk evaluation is performed on a multidisciplinary basis.

\section{ACKNOWLEDGEMENTS}

Funding. No funding or sponsorship was received for this study or publication of this article.

Authorship. All named authors meet the International Committee of Medical Journal Editors (ICMJE) criteria for authorship for this article, take responsibility for the integrity of the work as a whole, and have given their approval for this version to be published.

Disclosures. Riccardo CompagnonI, Roberta GualtierottI and Pietro Randelli have nothing to disclose.

Compliance with Ethics Guidelines. This article is based on previously conducted studies and does not contain any studies with human participants or animals performed by any of the authors.

Open Access. This article is distributed under the terms of the Creative Commons Attribution-NonCommercial 4.0 International License (http://creativecommons.org/licenses/ by-nc/4.0/), which permits any noncommercial use, distribution, and reproduction in any medium, provided you give appropriate credit to the original author(s) and the source, provide a link to the Creative Commons license, and indicate if changes were made.

\section{REFERENCES}

1. Gabriel SE, Michaud K. Epidemiological studies in incidence, prevalence, mortality, and comorbidity of the rheumatic diseases. Arthritis Res Ther. 2009;11(3):229.

2. Mertelsmann-Voss C, Lyman S, Pan TJ, Goodman S, Figgie MP, Mandl LA. Arthroplasty rates are increased among US patients with systemic lupus erythematosus: 1991-2005. J Rheumatol. 2014;41(5):867-74.

3. Jamsen E, Virta LJ, Hakala M, Kauppi MJ, Malmivaara A, Lehto MU. The decline in joint replacement surgery in rheumatoid arthritis is associated with a concomitant increase in the intensity of anti-rheumatic therapy: a nationwide register-based study from 1995 through 2010. Acta Orthop. 2013;84(4):331-7.

4. Au K, Reed G, Curtis JR, et al. High disease activity is associated with an increased risk of infection in patients with rheumatoid arthritis. Ann Rheum Dis. 2011;70(5):785-91.

5. Zhu Y, Zhang F, Chen W, Liu S, Zhang Q, Zhang Y. Risk factors for periprosthetic joint infection after total joint arthroplasty: a systematic review and meta-analysis. J Hosp Infect. 2015;89(2):82-9.

6. Gualtierotti R, Parisi M, Ingegnoli F. Perioperative management of patients with inflammatory rheumatic diseases undergoing major orthopaedic surgery: a practical overview. Adv Ther. 2018;35(4):439-56.

7. Pereira LC, Kerr J, Jolles BM. Intra-articular steroid injection for osteoarthritis of the hip prior to total hip arthroplasty: is it safe? A systematic review. Bone Jt J. 2016;98-B(8):1027-35.

8. Gualtierotti R. Understanding cardiovascular risk in rheumatoid arthritis: still a long way to go. Atherosclerosis. 2017;256:123-4.

9. Agca R, Heslinga SC, Rollefstad S, et al. EULAR recommendations for cardiovascular disease risk management in patients with rheumatoid arthritis and other forms of inflammatory joint disorders: 2015/2016 update. Ann Rheum Dis. 2017;76(1):17-28. 
10. Trevisan C, Klumpp R, Compagnoni R. Risk factors in transient osteoporosis: a retrospective study on 23 cases. Clin Rheumatol. 2016;35(10):2517-22.

11. Eka A, Chen AF. Patient-related medical risk factors for periprosthetic joint infection of the hip and knee. Ann Transl Med. 2015;3(16):233.

12. Symmons DPM, Gabriel SE. Epidemiology of CVD in rheumatic disease, with a focus on RA and SLE. Nat Rev Rheumatol. 2011;7(7):399-408.

13. Douketis JD, Spyropoulos AC, Spencer FA, Mayr M, Jaffer AK. Perioperative management of antithrombotic therapy: antithrombotic therapy and prevention of thrombosis, 9th edn: American College of Chest Physicians evidence-based clinical practice guidelines. Chest. 2012;141(2 Suppl):e326S-e350S.

14. Centola M, Cavet G, Shen Y, et al. Development of a multi-biomarker disease activity test for rheumatoid arthritis. PLoS One. 2013;8(4):e60635.

15. Curtis JR, Xie F, Chen L, Saag KG, Yun H, Muntner P. Biomarker-related risk for myocardial infarction and serious infections in patients with rheumatoid arthritis: a population-based study. Ann Rheum Dis. 2018;77(3):386-92.

16. Munroe ME, Vista ES, Guthridge JM, Thompson LF, Merrill JT, James JA. Proinflammatory adaptive cytokine and shed tumor necrosis factor receptor levels are elevated preceding systemic lupus erythematosus disease flare. Arthritis Rheumatol. 2014;66(7):1888-99.

17. Zoller B, Li X, Sundquist J, Sundquist K. Autoimmune diseases and venous thromboembolism: a review of the literature. Am J Cardiovasc Dis. 2012;2(3 SRC-BaiduScholar):171-83.

18. Oberweis BS, Nukala S, Rosenberg A, et al. Thrombotic and bleeding complications after orthopedic surgery. Am Heart J. 2013;165(3):427-33.

19. Trevisan C, Klumpp R, Auriemma L, Compagnoni R. An algorithm for predicting blood loss and transfusion risk after total hip arthroplasty. Transfus Apher Sci. 2018;57:272-6.

20. Goodman SM, Mackenzie CR. Cardiovascular risk in the rheumatic disease patient undergoing orthopedic surgery. Curr Rheumatol Rep. 2013;15(9):354.

21. de Laat B, Derksen RH, Urbanus RT, de Groot PG. IgG antibodies that recognize epitope Gly40-Arg43 in domain I of beta 2-glycoprotein I cause LAC, and their presence correlates strongly with thrombosis. Blood. 2005;105(4):1540-5.
22. de Laat B, Pengo V, Pabinger I, et al. The association between circulating antibodies against domain I of beta2-glycoprotein I and thrombosis: an international multicenter study. J Thromb Haemost. 2009;7(11):1767-73.

23. Andreoli L, Nalli C, Motta M, et al. Anti-beta(2)glycoprotein I IgG antibodies from 1-year-old healthy children born to mothers with systemic autoimmune diseases preferentially target domain 4/5: might it be the reason for their 'innocent' profile? Ann Rheum Dis. 2011;70(2):380-3.

24. Goodman SM, Springer B, Guyatt G, et al. 2017 American College of Rheumatology/American Association of Hip and Knee Surgeons guideline for the perioperative management of antirheumatic medication in patients with rheumatic diseases undergoing elective total hip or total knee arthroplasty. Arthritis Rheumatol. 2017;69(8):1538-51.

25. Berbari EF, Osmon DR, Carr A, et al. Dental procedures as risk factors for prosthetic hip or knee infection: a hospital-based prospective case-control study. Clin Infect Dis . 2010;50(1):8-16.

26. Correa JD, Calderaro DC, Ferreira GA, Mendonca SM, Fernandes GR, Xiao E. Subgingival microbiota dysbiosis in systemic lupus erythematosus: association with periodontal status. Microbiome. 2017;5(1 SRC_-BaiduScholar):34.

27. Bai $\mathrm{M}$, Tomenson $\mathrm{B}$, Creed $\mathrm{F}$, et al. The role of psychological distress and personality variables in the disablement process in rheumatoid arthritis. Scand J Rheumatol. 2009;38(6):419-30.

28. Trevisan CL, Klumpp R, Recalcati W, Compagnoni R. Influence of personality psychology on outcome of total hip arthroplasty: a cross-sectional study on 69 patients. Musculoskelet Surg. 2015;99(3):231-6.

29. Syed Z, Rubinfeld I. Personalized risk stratification for adverse surgical outcomes: innovation at the boundaries of medicine and computation. Pers Med. 2010;7(6):695-701.

30. Ramiro S, Sepriano A, Chatzidionysiou K, et al. Safety of synthetic and biological DMARDs: a systematic literature review informing the 2016 update of the EULAR recommendations for management of rheumatoid arthritis. Ann Rheum Dis. 2017;76(6):1101-36.

31. Nishida K, Harada $R$, Nasu $Y$, et al. The clinical course of patients with rheumatoid arthritis who underwent orthopaedic surgeries under disease control by tofacitinib. Mod Rheumatol. 2018:1-3. https://doi.org/10.1080/14397595.2018.1427431

32. Grennan DM, Gray J, Loudon J, Fear S. Methotrexate and early postoperative complications in 
patients with rheumatoid arthritis undergoing elective orthopaedic surgery. Ann Rheum Dis. 2001;60(3):214-7.

33. Randelli P, Randelli F, Cabitza P, Vaienti L. The effects of COX-2 anti-inflammatory drugs on soft tissue healing: a review of the literature. J Biol Regul Homeost Agents. 2010;24(2):107-14.

34. Mochizuki T, Yano K, Ikari K, et al. Tramadol hydrochloride/acetaminophen combination versus non-steroidal anti-inflammatory drug for the treatment of perioperative pain after total knee arthroplasty: a prospective, randomized, open-label clinical trial. J Orthop Sci. 2016;21(5):625-9.

35. Ting S, Schug S. The pharmacogenomics of pain management: prospects for personalized medicine. J Pain Res. 2016;9:49-56.

36. Behrooz A. Pharmacogenetics and anaesthetic drugs: implications for perioperative practice. Ann Med Surg (Lond). 2015;4(4):470-4.

37. Umicevic Mirkov M, Coenen MJ. Pharmacogenetics of disease-modifying antirheumatic drugs in rheumatoid arthritis: towards personalized medicine. Pharmacogenomics. 2013;14(4):425-44.
38. Nikiphorou E, Carpenter L, Morris S, et al. Hand and foot surgery rates in rheumatoid arthritis have declined from 1986 to 2011, but large-joint replacement rates remain unchanged: results from two UK inception cohorts. Arthritis Rheumatol. 2014;66(5):1081-9.

39. Jones CW, Jerabek SA. Current role of computer navigation in total knee arthroplasty. J Arthroplasty. 2018;33(7):1989-93.

40. Szczech B, McDermott JD, Issa K, et al. Patientspecific instrumentation in total knee arthroplasty: what is the evidence? J Knee Surg. 2016;29(4):341-5.

41. Christ AB, Pearle AD, Mayman DJ, Haas SB. Roboticassisted unicompartmental knee arthroplasty: stateof-the art and review of the literature. J Arthroplasty. 2018;33(7):1994-2001.

42. Zhu S, Qian W, Jiang C, Ye C, Chen X. Enhanced recovery after surgery for hip and knee arthroplasty: a systematic review and meta-analysis. Postgrad Med J. 2017;93(1106):736-42.

43. Mocsai A, Kovacs L, Gergely P. What is the future of targeted therapy in rheumatology: biologics or small molecules? BMC Med. 2014;12:43. 